\title{
Cécile Oumhani : se confronter au monde (à travers l'écriture)
}

Propos recueillis par Karin Schwerdtner, Université Western Ontario

Depuis 1995, année de parution en France de son premier recueil de poèmes, À l'abside des hêtres, et de son premier recueil de nouvelles, Fibules sur fond de pourpre, Cécile Oumhani a fait paraître une dizaine d'ouvrages de poésie, notamment aux éditions Voix d'encre. Avec Une odeur de henné, paru pour la première fois en 1999, elle «tente » le roman. Parmi les œuvres romanesques qui suivent cette première, plusieurs ont été récompensées par des prix : à savoir, Le café d'Yllka (2008), prix littéraire de l'Association des Écrivains de langue française 2009 ; L'atelier des Strésor (2012), mention spéciale du prix franco-indien Gitanjali et prix de la Bastide 2013 ; et une réédition (2012) de Une odeur de henné, prix Grain de Sel 2013. Outre ces écrits, dont les plus récents sont parus aux éditions Elyzad en Tunisie, son œuvre comporte un essai À fleur de mots (2005), un recueil collectif À cinq mains (2007) écrit en collaboration avec Emna Belhaj Yahya, Rajae Benchemsi, Maïssa Bey et Leïla Sebbar, et des articles ou textes de revue comme «Tunisia, A Time of Uncertainty» (2013) publié sur le site des «Words Without Borders ». Vient tout juste de paraître, aux éditions Elyzad, Tunisie, carnets d'incertitude (2013).

En juin 2013, au moment où elle lisait les épreuves de ce dernier livre sur la Tunisie, Cécile Oumhani m'a fait l'honneur de me recevoir chez elle, en région parisienne. Mes questions pour l'auteure ont porté principalement sur ses écrits de prose, lesquels thématisent notamment l'exil, le déplacement, et la mise en contact de cultures différentes. Dans un premier temps, l'auteure a bien voulu définir avec moi le «désir», terme qui revient dans son œuvre, et notamment dans ses romans, pour ensuite me préciser le rapport qui existe, pour elle, entre le besoin (par exemple, de « faire voler en éclats la rigidité des codes ») et un certain renoncement. Se référant à son éthique littéraire et parfois à ses carnets d'incertitude à paraitre, elle s'est expliquée, dans un deuxième temps, sur ce qui lui paraît une obligation morale, celle de repousser ses propres limites pour écrire, par exemple, sur les périls de la guerre. Revendiquant le droit et le besoin de suivre la voie qui s'impose à elle, d'un livre à un autre, l'auteure commente les risques pris pour certains projets et son choix de continuer à publier chez Elyzad. Ainsi, j'ai bien compris, chez Cécile Oumhani, l'importance d'être engagée, celle de faire face au monde à travers l'écriture. ${ }^{1}$ 
Q : Dans votre œuvre entière, et en particulier dans votre dernier roman, L'atelier des Strésor (2012), l'idée du désir revient comme un leitmotiv. Comment comprenez-vous cette notion ?

$\mathbf{R}$ : Le désir est lié à la pulsion de vie, à l'élan qui nous pousse vers l'extérieur et nous propulse à la découverte du monde, de ceux qui l'habitent et de nous-mêmes. Mais son corollaire est aussi une part de renoncement, avec l'obligation d'accepter ce qui n'est pas de l'ordre du réalisable. Il y a effectivement une dialectique entre ce vers quoi tend le désir et ce qui est de l'ordre du possible. Pourrions-nous inventer ou créer, au sens le plus large du terme, si ce que nous rêvons n'excédait pas les limites auxquelles nous finissons toujours par nous heurter?

Q : Écrire, est-ce donc se heurter à certaines limites ?

$\mathbf{R}$ : Il y a toujours ce qu'on désirerait écrire - ce qu'on aimerait avoir écrit - et la réalité de ce qu'on arrive à faire. Par la lucidité du regard porté sur son propre texte, et le re/travail qu'il amène, on peut repousser ses limites et essayer d'approcher les horizons qu'on rêve de franchir, même si, à la fin, on est toujours confronté à la nécessité d'un certain renoncement.

D'ailleurs, si on avait atteint, avec un livre particulier, ce qu'on voulait écrire, si on avait atteint cet objectif, peut-être cesserait-on d'écrire.

Q : Ainsi, dans votre cas, est-ce parce qu'il n'est jamais tout à fait réalisé que votre désir d'écrire se renouvelle?

R : C'est aussi parce que, d'un livre à l'autre, je découvre autre chose encore ; je découvre ce que j'ai envie d'explorer et que je ne connaissais pas. Pour prendre l'exemple de L'atelier des Strésor, jamais je n'aurais pensé que j'écrirais un roman qui se déroule à Paris au dix-septième siècle. Quand on m'avait proposé une résidence d'écriture à Saint Quentin en Yvelines, la consigne était d'établir un lien avec le dix-septième siècle, avec le musée de Marly-le-Roy. J'avais totale liberté, mais il fallait qu'il y ait ce lien. Quelle aventure ! Ça m'a vraiment passionnée, au point qu'ayant fini le roman, je me suis dit que j'aurais peut-être envie d'en écrire un autre, où il y aurait une trame historique, mais dans l'aire méditerranéenne. Ainsi, quand on termine un manuscrit, il y a toujours un autre horizon d'attente qui s'ouvre et avec lui les prémices d'un livre à venir. 
Q : Dans À fleur de mots, vous parlez d'un désir particulier, ou plutôt d'un besoin, de s'effacer en écrivant. Pourriez-vous vous expliquer davantage sur ce mouvement d'effacement de soi ?

$\mathbf{R}$ : Pour arriver à comprendre au mieux ce qu'on explore en écrivant un roman, il importe de ne pas s'installer dans une sorte de projection sur ses personnages. Il y a lieu de s'effacer par rapport à eux. Par exemple, j'ai dû m'esquiver, moi et ce que je suis au quotidien, pour écrire $L e$ café d'Yllka. Il le fallait, pour tenter de cerner ce qu'avait pu être la vie de cette femme que j'avais croisée à l'aéroport de Budapest. Sinon comment aurais-je pu espérer comprendre pourquoi elle avait l'air désespérée lorsque je l'ai aperçue ? Si, en écrivant L'atelier des Strésor, j'ai pu pénétrer un peu l'univers d'Henry Strésor et de sa fille, c'est que j'ai passé plusieurs semaines où je ne pensais plus qu'à eux, où j'oubliais qui j'étais, où j'oubliais le siècle dans lequel je vis pour pénétrer le leur à travers l'écriture. Dans les quelques documents que j'ai pu consulter, j'ai vu que la femme d'Henry Strésor était décédée au début du mois de mai d'une année donnée. Quelques mois plus tard, précisément à la même date au mois de décembre, Henry mourait à son tour dans un accident de la circulation à Paris. Comment a-t-il vécu ce deuil au point que, le jour anniversaire de la mort de cette femme qu'il avait aimée, il n'a pas vu cette charrette, qu'il en a été renversé et qu'il en est mort ? Pour moi, ce n'était pas du hasard. Quelques traces laissées sur de vieilles pages ont suscité le besoin de les déchiffrer par le biais d'une autre écriture, qui chercherait à combler ces mots perdus, à élucider des phrases restées muettes.

L'écriture poétique nécessite l'effacement du «moi diurne » pour que puissent jaillir d'autres choses qu'on ne maîtrise pas et qui nous parcourent. Ces choses sont fragiles et fugaces comme un rêve qu'on ne prend pas la peine de noter immédiatement alors qu'on sort à peine du sommeil. Cela ne veut pas dire qu'après, on ne revient pas sur ce qu'on a écrit pour s'atteler au travail qu'est l'écriture. Il peut arriver qu'on écrive un passage auquel on ne changera rien du tout. Mais, pour cela, il faut cette première phase où on s'efface, où c'est presque l'inconscient qui parle, la part inconnue de soi-même. Il faut aussi un retour vers le texte, un retour critique qui va faire que soit on décide de ne rien changer, soit on décide d'élaguer, de réécrire. Il y a donc un mouvement de va et vient, de balancier entre ce qui vient de façon non contrôlée, un petit peu comme l'écriture automatique - pas tout à fait, mais quand même -, et puis au contraire une grande rigueur et du recul par rapport à ce qu'on écrit. 
Q : Dans L'atelier des Strésor, deux phrases m'ont particulièrement accrochée: « Il fallait que le monde se dévoile à lui, autre, multiple et chatoyant. Sinon il ne peindrait plus » (15). Peut-être avez-vous éprouvé parfois ce besoin ? Pour écrire, faut-il que le monde se dévoile à vous ainsi ? $\mathbf{R}$ : C'est vrai qu'il y a une force qui me pousse à découvrir. Sans elle, je n'aurais pas écrit ce que j'ai écrit. Je pense à cette femme que j'ai véritablement croisée à l'aéroport de Budapest. J'étais bouleversée par ce que je sentais, et je ne cessais pas d'y penser. Pour y parvenir, il fallait que j'arrive au moins à des hypothèses plausibles, concernant ce qui avait pu la mener à l'état où elle était ce matin-là.

Dans le cas de L'atelier des Strésor, ce qui m'incitait à entrer dans l'univers d'Henry Strésor et de sa fille, c'était le défi [proposé par la Maison de la Poésie de Saint-Quentin-enYvelines et le Musée-Promenade]. J'ai trouvé passionnant d'essayer de suivre cette contrainte jusqu'au bout, là où elle me menait. C'était stimulant de relever ce défi, de faire toutes les recherches nécessaires pour construire quelque chose qui soit cohérent. La contrainte peut être intéressante, voilà. C'est la conclusion que je tire de L'atelier des Strésor.

Q : En quoi la lecture aurait-elle stimulé, chez vous, ce que vous appelez, dans $\grave{A}$ fleur de mots, la « passion de l'écriture »?

$\mathbf{R}$ : Très tôt, la correspondance, les lettres reçues m'ont fait découvrir des lieux et des individus que je ne connaissais pas encore. Ces mots tracés à l'encre qui nous arrivaient du Canada anglophone, d'Ontario et de Colombie Britannique, où avait émigré la famille de ma mère étaient les seuls liens, la seule façon d'être en contact, de me représenter des personnes qui comptaient beaucoup pour elle et pour nous. En même temps, je lisais énormément. À ma sœur et moi, ma mère avait commencé à nous lire des livres à voix haute quand on était toutes petites. De cette manière, j'ai découvert assez tôt le pouvoir magique des mots et l'importance des livres. Aussi loin que je me souvienne, nous avons lu conjointement dans les deux langues de la maison, en anglais et en français.

L'écriture et la lecture sont des formes de voyage, j'en suis absolument convaincue. C'est ce que j'ai compris en ressassant la correspondance familiale, en m'immergeant dans les livres dont nous étions entourés à la maison. C'est un espace de liberté qu'on ne peut pas vous prendre, un espace qui peut transcender l'absence et offrir des réponses, lorsque le monde autour de vous ne peut ou ne veut pas vous les donner. Je pense qu'on peut satisfaire beaucoup de besoins grâce 
à ces espaces, qu'il s'agisse d'y accéder en tant qu'écrivain ou en tant que lecteur. D'ailleurs, dans quelle mesure le lecteur n'écrit pas lui aussi le texte ? Je le pense un peu.

Q : Lors d'un entretien avec Brigitte Aubonnet, vous racontez votre visite à Zagreb pour la parution d'une traduction des Racines $d u$ mandarinier (2001), et vous dites avoir été très interpellée de voir qu'on s'intéressait à votre livre et au message de tolérance qu'on y lisait...

$\mathbf{R}$ : Le message, on ne doit pas en être trop conscient au moment où on écrit. On ne devrait pas en être trop conscient. Le risque est grand d'arriver à un livre qui n'est pas un roman mais qui relève plutôt du manifeste ou du pamphlet, ce qui n'est pas la même chose. C'est au lecteur d'enrichir le texte, ou, en quelque sorte, de l'écrire lui-même, dans ce qu'il apporte avec son propre regard, sa propre mémoire, ses propres expériences. C'est Vénus Khoury-Ghata qui dit qu'un livre vit sa vie et que sa vie nous échappe. Personnellement, j'aime bien cette idée qu'un texte nous échappe. Il nous échappe parce que les lecteurs se le réapproprient et puis qu'il va cheminer d'un endroit à l'autre. Ce cheminement, on ne le contrôle pas. Et donc, si l'auteur s'efface, c'est peut-être pas mal dans ce sens-là, aussi.

Les lecteurs peuvent effectivement vous enrichir avec leur regard. Ça a été le cas à propos du Café d'Yllka. En 2009, lors de 1'Escale du livre à Bordeaux, j'ai appris qu'en exYougoslavie, il y avait un poème devenu chanson, « Emina », que tous chantaient. Sans que j'aie pu le préméditer, le personnage de mon roman rappelle, par son prénom, cette «Emina » du célèbre poème d'amour d'Aleksa Šantić (1868-1924). Par la suite, j'ai pu en lire la traduction sur internet et me rendre compte que ce qu'il dit va bien avec Le café d'Yllka... Oui, les livres sont de fabuleux espaces de voyages et de rencontres, qui s'accroissent aussi du vécu, des souvenirs, de la culture de ceux qui les lisent.

Q : Ma prochaine question porte sur ce que vous appelez, chez l'écrivain, le besoin « de faire voler en éclats la rigidité des codes » (À fleur de mots 71). Quel est ce désir de s'opposer aux codes ? Pour en faire état, quelles sont, à votre avis, la littérature et la langue les plus adaptées ? R : Il y a cette notion de musicalité qui fait que, lorsque j'écris, j'entends ma phrase, qu'il s'agisse d'un roman ou d'un poème. Il faut que je la recompose jusqu'au moment où j'ai l'impression que c'est à peu près juste ; et ce « juste », je ne peux pas toujours l'expliquer.

Il y a donc cette première chose. Mais ce que j'ai vécu avec la révolution en Tunisie - 
c'est le livre qui va sortir au mois d'octobre [Tunisie, carnets d'incertitude] - peut sans doute être intéressant pour la question que vous posez. La situation était telle que je ne pouvais plus écrire comme j'avais écrit auparavant. Je ne pouvais plus écrire de roman. La réalité dehors dépassait toute fiction. Il était pour moi impossible de me mettre en retrait par rapport à ce qui se passait à l'extérieur et qui me sollicitait heure par heure. Même pour la langue elle-même, je ne pouvais plus du tout utiliser les mêmes images. En revanche, je pouvais écrire des fragments, des choses qui me touchaient fortement, en écho avec ce que j'entendais, avec ce que je voyais. Le rythme des slogans scandés par les foules chaque jour dans toute une région du monde me traversait jusque dans mon sommeil. Ce n'était plus du tout la musique entendue lors de mes précédents livres, c'était quelque chose de beaucoup plus haché, dépouillé d'adjectifs et d'adverbes. Des phrases, même des phrases nominales. Oui, c'est un rythme tout à fait différent qui s'est imposé sur la page. J'en suis donc convaincue : la conception de l'écriture évolue en fonction des circonstances, même si je garde une exigence par rapport aux sonorités. Quand il y a des gens qui meurent tous les jours, on ne peut plus entendre la même musique, c'est impossible. Impossible d'écrire les mêmes textes et d'adopter la même écriture.

Q : Cette expérience, croyez-vous qu'elle a changé votre écriture pour toujours ?

$\mathbf{R}$ : Il me semble que, depuis la révolution tunisienne, mon autocensure est tombée...

Q : Votre autocensure?

R: Je pense qu'il est important d'être audacieux; de se lancer vers l'extérieur; de suivre jusqu'au bout, avec exigence, avec authenticité, avec sincérité, ce qu'on a envie d'écrire.

Q : À ce sujet de ce qui est audacieux : évoquer les périls de la guerre, comme vous le faites, par exemple, dans Le café d'Yllka et L'atelier des Strésor, est-ce, pour vous, un acte risqué ?

$\mathbf{R}$ : Cet acte, je l'ai pensé plutôt comme une nécessité, comme une obligation morale. On ne peut pas fermer les yeux, prétendre ignorer. Il y a pour moi une immoralité absolue à se lever le matin comme si de rien n'était, à boire son café et à manger des loukoums, comme on est en train de le faire en ce moment, alors qu'il y a des familles entières qui sont massacrées en Syrie et sans doute dans d'autres pays encore. Une immoralité constitutive de notre condition d'êtres humains, enfermés, séparés par les distances, les structures de pouvoir qui régissent le monde... Et je 
refuse de dire « on n'y peut rien », tout en sachant que ce qu'on peut semble parfois minuscule, insignifiant. N'est-ce pas dans l'énergie de ces refus, si minuscules soient-ils, si insignifiants semblent-ils que l'on peut tenter d'avancer et de faire changer les choses ? Pour moi, l'écriture est aussi une manière de résister, de dire non à cette gangrène qui nous endort, qui émousse nos révoltes, et étouffe nos cris de scandale.

Q : Si l'écriture est une façon de résister, n'est-elle pas, aussi, une prise de risque ? Un défi ?

$\mathbf{R}$ : Avec Le café d'Yllka, j'essayais d'écrire sur une guerre que je n'avais pas vécue. Comment écrivez-vous une guerre que vous n'avez pas vécue ? Quelle légitimité avez-vous à le faire? Quels personnages allez-vous choisir pour arriver à le faire ? Finalement, le personnage de jeune fille avec ce retour sur l'enfance - avec ce côté universel d'un regard d'enfant dans ce qu'il a d'innocent, parce qu'il ne connaît pas encore -, me permettait de me frayer un chemin dans des territoires que je connaissais seulement à travers les poètes que j'avais rencontrés dans des festivals de poésie en Europe centrale et qui avaient vécu ces guerres et qui m’en avaient parlé, ou les paysages que j'ai traversés avec eux et qui portaient encore les marques de la guerre.

Le prochain texte qui va paraître en octobre aux éditions Elyzad [Tunisie, carnets d'incertitude], est constitué de fragments qui concernent la Tunisie dans la période 2011-2013. J'y utilise un « je » qui correspond à ma propre expérience ; j’y relate, de manière fragmentaire, sans chronologie stricte, des choses que j’ai vraiment vécues...

Q : Que peut-il y avoir de dangereux ou risqué à écrire sur le printemps arabe en Tunisie ?

R : Pas grand chose pour moi qui vis en France, de l'autre côté de la Méditerranée... C'est certainement différent pour les artistes, les écrivains, les journalistes qui vivent en Tunisie et doivent faire face à la menace de groupes salafistes extrémistes qui sévissent ici et là dans le pays. Des plasticiens ont pu s'en rendre compte en juin 2012 lors du Printemps des Arts de La Marsa, lorsqu'ils ont été attaqués physiquement parce que les œuvres exposées déplaisaient à ces obscurantistes. La projection de certains films comme Persépolis ou Laïcité Inchallah ont déchaîné une violence inouïe. Des sites de journaux, d'éditeurs ont été récemment victimes de cyber-attaques perpétrées par des groupes de cette mouvance. Des musiciens ont pu s'en rendre compte il n'y a pas longtemps, arrêtés, incarcérés par le gouvernement parce que leurs chansons étaient jugées irrévérencieuses. Des cinéastes aussi... La liberté d'expression, l'un des mots 
d'ordre de la révolution de janvier 2011, n'est pas encore un acquis. Elle est encore à asseoir, à défendre contre tout ce qui la menace à présent.

Q : Selon vous, où réside le plus grand risque pour l'écrivain désireux de résister par les mots ? Pour vous, auteur tout dernièrement des Carnets d'incertitude, où réside le plus grand danger? Est-ce dans le choix, par exemple, d'un éditeur et d'un pays d'édition?

$\mathbf{R}$ : Il est vrai que c'est une question importante lorsqu'on entre en écriture. On devient associé à un catalogue, à l'image donnée par une maison d'édition. Trouver un éditeur n'est pas forcément une chose facile, et c'est un long cheminement que de trouver la maison d'édition avec laquelle on a des affinités profondes et puis d'être accepté par elle, d'y construire ce qui est pour moi doit se fonder sur une relation humaine.

On pourrait se demander pourquoi je publie mes livres en Tunisie alors que je vis en France : au début, c'était peut-être un peu ma manière de trouver une forme de soulagement à l'exil. Or depuis la révolution tunisienne, rester chez Elyzad est, pour moi, une forme d'engagement. Il me tient vraiment à cœur de continuer de cheminer avec Élisabeth Daldoul. Justement, j'ai l'impression que rester chez mon éditrice, c'est une forme de résistance - par rapport à des façons de voir qu'on a ici, et par rapport aux dérives de la révolution tunisienne en ce moment. Enfin, ce n'est peut-être pas souvent qu'on a l'occasion de travailler avec un éditeur qui fait le travail qu'elle fait avec autant d'exigence et d'humanité aussi.

Il me semble qu'un éditeur n'est pas là seulement pour imprimer votre livre, il est là aussi pour le lire, pour le regarder en détail et pour dialoguer avec l'auteur. Il doit y avoir un échange. C'est ça, le travail d'un éditeur. Il s'agit de faire vivre le livre, de l'accompagner. Et ce sont des tâches dont mon éditrice et ceux qui travaillent avec elle s'acquittent admirablement.

Q : De votre côté, vous l'avez revendiqué : il vous fait plaisir de découvrir, avec chaque projet de livre, ce que vous ne connaissiez pas encore. Pour vous, le changement de voie, d'un livre à l'autre, ne pose donc pas de problème ? Il ne représente pas pour vous un danger?

$\mathbf{R}$ : Après la publication de mon roman L'atelier des Strésor, certains lecteurs ont été déroutés, surpris, parce que j'avais écrit des romans qui avaient un univers méditerranéen et que subitement, un de mes romans se passait à Paris au dix-septième siècle. Mais je crois qu'au-delà des différences qui séparent un livre du suivant, ils sont intimement liés les uns aux autres. C'est 
parce que j'ai écrit ce roman situé au dix-septième siècle que je pourrai peut-être en écrire un autre qui aura aussi une trame historique. C'est parce qu'un roman m'a emmenée à Zagreb quand il a été traduit, parce que mes poèmes m'ont emmenée dans les Balkans pour des festivals que j'ai écrit Le café d'Yllka. L'écriture d'un livre nous change en profondeur par l'aventure de l'écriture elle-même et aussi par les rencontres et les voyages qu'il entraîne. Le changement de voie est seulement une apparence. Il n'est possible que parce les autres livres l'ont précédé. Un livre porte les germes du suivant pour peu que l'on soit attentif à ce que nous ouvre l'écriture à un moment donné. Et c'est passionnant de se mettre ainsi à l'écoute de ce qui viendra peut-être par la suite parce qu'on n'est jamais sûr de rien, parce qu'il serait présomptueux d'avoir trop de certitudes.

Je revendique le droit et le besoin de suivre la voie qui s'impose à moi d'un livre à un autre, quels que soient les changements d'univers, d'époque ou de lieu, d'écriture aussi. Je n'ai pas envie de me répéter, dans la mesure du possible. Je n'ai pas envie de me laisser enfermer dans l'image d'une écrivaine qui écrirait sur les femmes entre guillemets, sur le Maghreb. Non, pour moi l'écriture, c'est un territoire universel, et j'éprouve parfois la nécessité de changer d'univers. L'écriture est un cheminement fait de voyages et de traversées qui doivent s'accomplir, pour qu'elle se poursuive, pour qu'elle évolue.

Avec Le café d'Yllka, j'avais complètement quitté le Maghreb. Avec L'atelier des Strésor, j'ai quitté à la fois Le café d'Yllka et les univers précédents, pour explorer une nouvelle époque et un nouveau lieu. En même temps, on y retrouve des sujets qui me sont chers : l'exil, la peinture, la question des femmes... On ne peut pas non plus se renouveler complètement, en tout cas ce n'est pas mon cas pour le moment, plus modestement. Le peut-on ? Echappe-t-on à soimême, à ce qui nous hante en écrivant? Je vous parlais de la nécessité d'un effacement de soi pour écrire. Cet effacement relève d'une mise à distance pour aller vers l'autre. Mais je ne pense pas qu'on aille vers l'autre à contre-courant de ce que l'on est ou de la part obscure de nousmêmes? Alors même que je cherche à sonder des personnages et des univers, je le fais à travers le prisme de mon regard. Et la nouveauté d'un livre n'élude pas la présence des thèmes, de refrains qui courent d'un texte à un autre. 
Q : Et avec votre prochain livre?

$\mathbf{R}$ : Je suis impatiente de repartir dans l'écriture d'un roman où il y aura une trame historique et où je vais revenir un petit peu vers la Méditerranée mais aller aussi vers l'Amérique du Nord. Il s'agira d'embrasser quelque chose de plus universel. Je ne peux pas en dire plus pour le moment.

\section{Référence}

Aubonnet, Brigitte. « Cécile Oumhani»(entretien). Encres Vagabondes. http://www.encresvagabondes.com/rencontre/oumhani.htm (consulté le 8 novembre 2013).

\section{Textes cités de Cécile Oumhani}

À cinq mains, avec Emna Belhaj Yahya, Rajae Benchemsi, Maïssa Bey et Leïla Sebbar. Tunis : Elyzad, 2007.

À fleur des mots. La passion de l'écriture. Montpellier/Blida : Chèvrefeuille étoilée, 2004.

À l'abside des hêtres. Valenciennes : Centre Froissart, 1995.

Cités d'oiseaux (2011).

Fibules sur fond de pourpre. Solignac : Le Bruit des autres, 1995.

L’atelier des Strésor. Tunis : Elyzad, 2012.

Le café d'Yllka. Tunis : Elyzad, 2008.

Les racines du mandarinier. Paris : Paris-Méditerranée, [2001] ; Korijeni mandarine (traduction en croate). Zagreb : Ljevak, 2007.

« Tunisia, A Time of Uncertainty ». Words Without Borders, mars 2013. http://wordswithoutborders.org/dispatches/article/tunisia-a-time-of-uncertainty

Tunisie, carnets d'incertitude. Tunis : Elyzad, 2013.

Une odeur de henné. Paris : Paris-Méditerranée, 1999 ; Tunis : Elyzad, 2012.

NOTE de l'auteure Cécile Oumhani

${ }^{1}$ La nouvelle constitution tunisienne a été votée le 26 janvier 2014, marquant un tournant décisif dans le processus de transition démocratique en cours dans le pays depuis la révolution de 2011. Tout en étant conscients que des enjeux importants restent devant eux (code électoral, élections à venir...), les Tunisiens sont moins crispés et moins inquiets qu'en 2013. 